\title{
Atomic Mutagenesis of the Ribosome: Towards a Molecular Understanding of Translation
}

\author{
Norbert Polacek*
}

\begin{abstract}
The multifaceted repertoire of non-protein-coding RNAs (ncRNAs) in organisms of all three domains of life emphasizes their fundamental role in biology. Research in my lab focuses on revealing the regulatory and catalytic function of small and large ncRNAs in different model organisms. In particular we are interested in understanding ncRNA/protein complexes such as the vault complex or the ribosome. The ribosome, the central enzyme of protein biosynthesis, is a multifunctional ribonucleoprotein particle composed of two unequal subunits that translates the genome's message into all proteins needed for life. The crucial role the translation machinery plays in gene expression is also mirrored by the fact that the ribosome represents the main target for antibiotics. Decades of genetic, biochemical and recent crystallographic studies revealed the ribosome as an RNA-enzyme with roots in the 'RNA world'. Despite these experimental insights, the catalytic and regulatory mechanisms of the ribosomal RNA are still not fully understood at the molecular level. To unravel the detailed contributions of rRNA nucleotides for protein synthesis we have developed and applied an 'atomic mutagenesis' approach. This tool allows the role of specific $23 \mathrm{~S}$ rRNA functional groups and even individual atoms to be studied during various stages of the ribosomal elongation cycle with thus far unequalled precision. This experimental approach bridges the disciplines of biochemistry and organic chemistry and has recently revealed specific functional $23 S$ rRNA groups involved in peptide bond synthesis, peptidyl-tRNA hydrolysis, GTPase activation, and tRNA translocation.
\end{abstract}

Keywords: Nucleotide analog interference · Protein synthesis · Ribosome · RNA structure and function

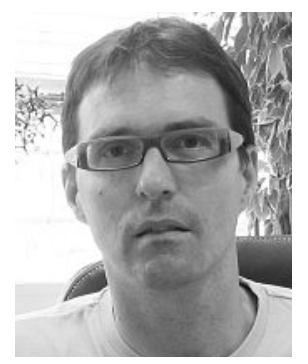

Norbert

studied biology/genetics at the University of Vienna, Austria, where he received his diploma in 1996 and his doctoral degree in biochemistry in 2000. This was followed by three years of postdoctoral studies at the Center for Pharmaceutical Biotechnology at the University of Illinois at Chicago. In 2003 he returned to Europe and accepted an assistant professor position at the Division of Genomics and RNomics at the Medical University Innsbruck. Sponsored by the START award of the Austrian Science Foundation he established his independent research group and received his habilitation (venia

\footnotetext{
*Correspondence: Prof. Dr. N. Polacek University of Bern

Department of Chemistry and Biochemistry

Freiestrasse 3

$\mathrm{CH}-3012$ Bern

Tel.: +41316314320

E-mail: norbert.polacek@dcb.unibe.ch
}

docendi) in molecular biology in 2005. He joined the Department of Chemistry and Biochemistry at the University of Bern in January 2012 as full professor of biochemistry. The focus of his research centers on the catalytic and regulatory potential of non-protein-coding RNA molecules in model organisms spanning all three domains of life. Online information about the Polacek group at the University of Bern can be found at http://polacek.dcb. unibe.ch/

\section{The Ribosome - An Ancient Enzyme Powered by RNA Catalysis}

The ribosome is a multifunctional RNA-protein complex and translates the genome's message into all proteins needed for life. Translation of the genetic information represents the final step in gene expression. The crucial role of protein biosynthesis in gene expression is highlighted by the fact that the ribosome represents the main target for clinically relevant antibiotics. Therefore the knowledge of its functioning is crucial for our understanding of antibiotic resistance and for the future design of new anti-microbial compounds. The ribosome is the largest known RNA enzyme and is regarded as one of the most ancient catalysts in biology. The bacterial ribosome has a molecular weight of
2.6-2.8 MDa with about $2 / 3$ of the mass comprised of ribosomal RNA (rRNA) and $1 / 3$ of ribosomal proteins ( $r$-proteins). The ribosome is a highly dynamic particle and can be regarded as a molecular machine consisting of two unequally sized subunits. The large 50S subunit contains two rRNA molecules (the 2'900 nucleotide-long 23S rRNA and the 120 residue-long 5S rRNA) and about 33 different r-proteins. The small 30S subunit on the other hand carries a single rRNA strand (the $\sim 1 ' 500$ nucleotide-long 16S rRNA) and approximately 20 r-proteins. All ribosomes have three distinct tRNA binding sites designated the A-site (aminoacyl-tRNA or acceptor site), P-site (peptidyl-tRNA or donor site), and E-site (tRNA exit site).

The catalytic center of the ribosome is located on the 50S subunit where the individual amino acid monomers are chemically linked via peptide bonds to form the polypeptide chain. This active site is referred to as the peptidyl transferase center (PTC) and it is located in a funnelshaped deep cleft on the interface side of the 50S subunit. Peptide bond synthesis and peptidyl-tRNA (pept-tRNA) hydrolysis are the two central chemical reactions of protein synthesis that take place in the PTC (for reviews see refs. [1,2]). Peptide bond formation involves aminolysis by the $\alpha$-amino group of the A-site bound aminoacyl-tRNA (aa-tRNA) of the ester 
bond that carries the nascent peptide at the terminal ribose C3' position of pept-tRNA. Subsequent to the nucleophilic attack of the $\alpha$-amino group, a short-lived tetrahedral transition state is formed that breaks down by donating a proton to the leaving oxygen to yield the reaction products deacylated tRNA at the P-site and pepttRNA (elongated by one amino acid) at the A-site. From an energetic point of view formation of a peptide bond is a downhill reaction since almost $8 \mathrm{kcal} / \mathrm{mol}$ are 'stored' in the ester bond of aa-tRNA and only $\sim 0.5 \mathrm{kcal} / \mathrm{mol}$ are needed for amide bond formation. Nevertheless, the uncatalyzed reaction (extrapolated from model reactions) occurs very slowly in solution with less than one bond formed per day. ${ }^{[3]}$ Thus the ribosome accelerates the rate of peptide bond formation approximately $10^{7}$-fold. ${ }^{[4]}$ The mechanism by which the PTC accelerates peptide bond formation has long been a question of rather intense discussions over decades (reviewed in ref. [1]). Before the discovery of the first RNA enzymes an r-protein-based catalytic mechanism was proposed even though it had already been realized that the function of rRNA inside the ribosome exceeds that of a mere scaffold for the r-proteins. The debates whether or not the ribosome is a ribozyme sustained until the beginning of the new millennium when high-resolution crystallographic structures ended these disputes by revealing the inner shell of the PTC as an all-RNA active site. ${ }^{[5,6]}$ Five universally conserved residues of domain $\mathrm{V}$ of $23 \mathrm{~S}$ rRNA form the catalytic crevice. From an evolutionary point of view it is remarkable that the ribosome, so to say the mother of all contemporary protein enzymes, is an RNA enzyme. Obviously the PTC proved itself as a very efficient RNAbased catalyst and has therefore not been replaced by proteins during the course of evolution. The ribosome can thus be regarded as molecular relic that outlived the transition from the 'RNA world' to contemporary biology.

\section{Making Proteins: The Ribosomal Elongation Cycle}

Protein biosynthesis can be divided into four different steps: initiation, elongation, termination and recycling. ${ }^{[7]}$ During the elongation cycle of translation, the tRNAs have to move along a $100 \AA$ path through the ribosome in steps of $10-30 \AA$. The initiator tRNA (fMet-tRNA ${ }^{\mathrm{fMet}}$ in bacteria) is delivered to the $30 \mathrm{~S}$ particle as ternary complex with initiation factor 2 (IF2) and GTP, where it binds to the AUG mRNA start codon which is displayed in the 30S P-site with the aid of IF1 and IF3. Subsequently the 50S subunit joins which results in triggering of the GTPase activity of IF2 and subsequent dissociation of the IFs from the 70S initiation complex. During the elongation phase of translation, the next amino acid is delivered to the A-site in form of the ternary complex composed of aa-tRNA, elongation factor Tu (EF-Tu) and GTP. Accuracy of translation is maintained in the $30 \mathrm{~S}$ decoding center by a sophisticated mechanism relying on monitoring the shape of the codon-anticodon mini-helix as well as the conformation of the tRNA anticodon stem-loop by highly conserved $16 \mathrm{~S}$ rRNA elements. ${ }^{[8,9]}$ For correct decoding only exact WatsonCrick interactions between the mRNA codon and the tRNA anticodon (primarily monitored at the first two nucleotides of the codon) are allowed and trigger, by a so far not fully understood signaling pathway mechanism, GTP hydrolysis on EF-Tu. EF-Tu•GDP subsequently dissociates from the aa-tRNA. The acceptor end of aa-tRNA subsequently swings into the 50S A-site, a process called accommodation. Also during this accommodation step discrimination between correct and incorrect aa-tRNAs can be achieved, since it has been shown that the rate of accommodation for cognate aa-tRNA is significantly faster than for near-cognate aa-tRNAs and thus the latter can even be rejected at this stage. ${ }^{[10]}$ Once the CCA terminal acceptor end of the aa-tRNA is fully accommodated in the PTC, peptide bond formation occurs immediately. While catalyzing 15 to 20 peptide bonds per second ${ }^{[11,12]}$ the error rate remains in the range of $10^{-3}$ to $10^{-4} \cdot[10,13,14]$ During the course of peptide bond formation the peptidyl moiety of P-site located pept-tRNA is transferred to the aa-tRNA in the A-site. This results in the elongation of the growing peptide chain by one amino acid at the C-terminal end. The nascent peptide departs the catalytic center via the so-called peptide exit tunnel, a 100 ̊ long and rRNA-rich path that spans the entire $50 \mathrm{~S}$ subunit and has its end at the solvent side. In the final step of the elongation cycle the deacylated tRNA and the pept-tRNA are translocated from the $\mathrm{P}$ - and A-sites to the E- and P-sites, respectively. This wellorchestrated tRNA/mRNA movement is promoted by the action of EF-G and GTP hydrolysis, which results in the entrance of a new mRNA codon into the decoding center of the small ribosomal subunit A-site. At the end of the open reading frame an mRNA stop codon (UAA, UAG, or UGA) is moved into the A-site. This results in the establishment of the termination phase of protein biosynthesis. Stop codons are usually not recognized by tRNA anticodons but by specific peptide motifs of class I release factors (RF1 and RF2 in bacteria). Binding of a class I RF to the A-site of the ribosome results in the hydrolysis of the ester bond that connects the fully synthesized peptide chain and the tRNA in the PTC P-site. This reaction is the second chemical reaction that is catalyzed in the PTC during protein synthesis. A universally conserved GGQ tri-peptide motif, which is located in a flexible loop of the RF, reaches into the PTC where it contacts and repositions conserved 23S rRNA residues, such as the crucial nucleotide A2602.[15,16] These structural changes and interactions with PTC nucleotides are thought to be critical for the GGQ motif to adopt its functional conformation and allows it to coordinate the hydrolytic water molecule that finally cleaves the pept-tRNA ester bond.[17] Finally, the recycling phase of translation frees the ribosome from the mRNA, the tRNAs and the class I RF. The latter are removed from the ribosome by the release factor RF3, which represents another translational GTPase. Before the ribosome can re-initiate at another mRNA molecule it needs to be dissociated into its subunits. This is established via binding of the ribosomal recycling factor RRF and EF-G GTPase activity. ${ }^{[7]}$

\section{Nucleotide Analog Interference in the Ribosome Using the Atomic Mutagenesis Approach}

While the crystal structures and cryoEM images of ribosomal complexes provide a wealth of detailed insights, they do not reveal definite answers about the molecular mechanisms of ribosome-triggered reactions. For example, despite the structural identification of the inner shell 23S rRNA nucleotides that line up the catalytic center of the ribosome, the detailed mechanism how peptide bonds are formed remains controversial and far from being understood at the molecular level. [1,2,18-22] Mutational studies of PTC nucleotides turned out to be insufficient to unequivocally disclose the contribution of specific 23S rRNA functional groups to translation at the atomic level..23] It appears that the level of chemical engineering that can be generated via standard mutagenesis is not adequate since it is limited to only three possible nucleobase changes that can be introduced by RNA polymerase. [24]

To circumvent these limitations and to be able to fully use the structural information provided by crystallographic and cryoEM studies, we have established an in vitro genetics approach allowing the site-directed manipulation of 23S rRNA residues in the context of the 50S ribosomal subunit at the functional group or even single atom level.[25,26] This approach permits changing the chemical characteristic of a specific residue within an RNA molecule containing thousands of nucleotides. The key fea- 
ture of this approach is the use of circularly permuted (cp) 23S rRNA transcripts as the major component for in vitro reconstitution of 50S particles (see Fig. 1 for details). The generation of a cp-23S rRNA is possible without dramatic structural consequences due to the proximity of the natural 5' and 3' ends, which form helix 1 (H1) of native 23S rRNA (Fig 1). The new ends of the cp-23S rRNA are placed such that a short sequence gap is introduced (typically between 25 and 55 nucleotides), which encompasses the 23S rRNA residue under investigation. The missing rRNA segment is chemically synthesized to either contain the wild type sequence, or non-natural nucleoside analogs. During an in vitro reconstitution procedure this synthetic
RNA piece is assembled with all the other components of the 50S subunit to form a large ribosomal subunit. Depending on the studied ribosomal function, the chemically engineered 50S subunit can subsequently be joined with native $30 \mathrm{~S}$ subunits to yield a complete $70 \mathrm{~S}$ ribosome for functional analyses. Thus with this experimental design, which we dubbed 'atomic mutagenesis', individual functional groups or even single atoms on 23S rRNA residues can be exchanged within the context of the whole $70 \mathrm{~S}$ ribosome.

The atomic mutagenesis technique has so far been successfully applied to study peptide bond formation, ${ }^{[26-28]}$ release factor-mediated peptidyl-tRNA hydrolysis, ${ }^{[15]}$ ribosome-triggered activation of transla-

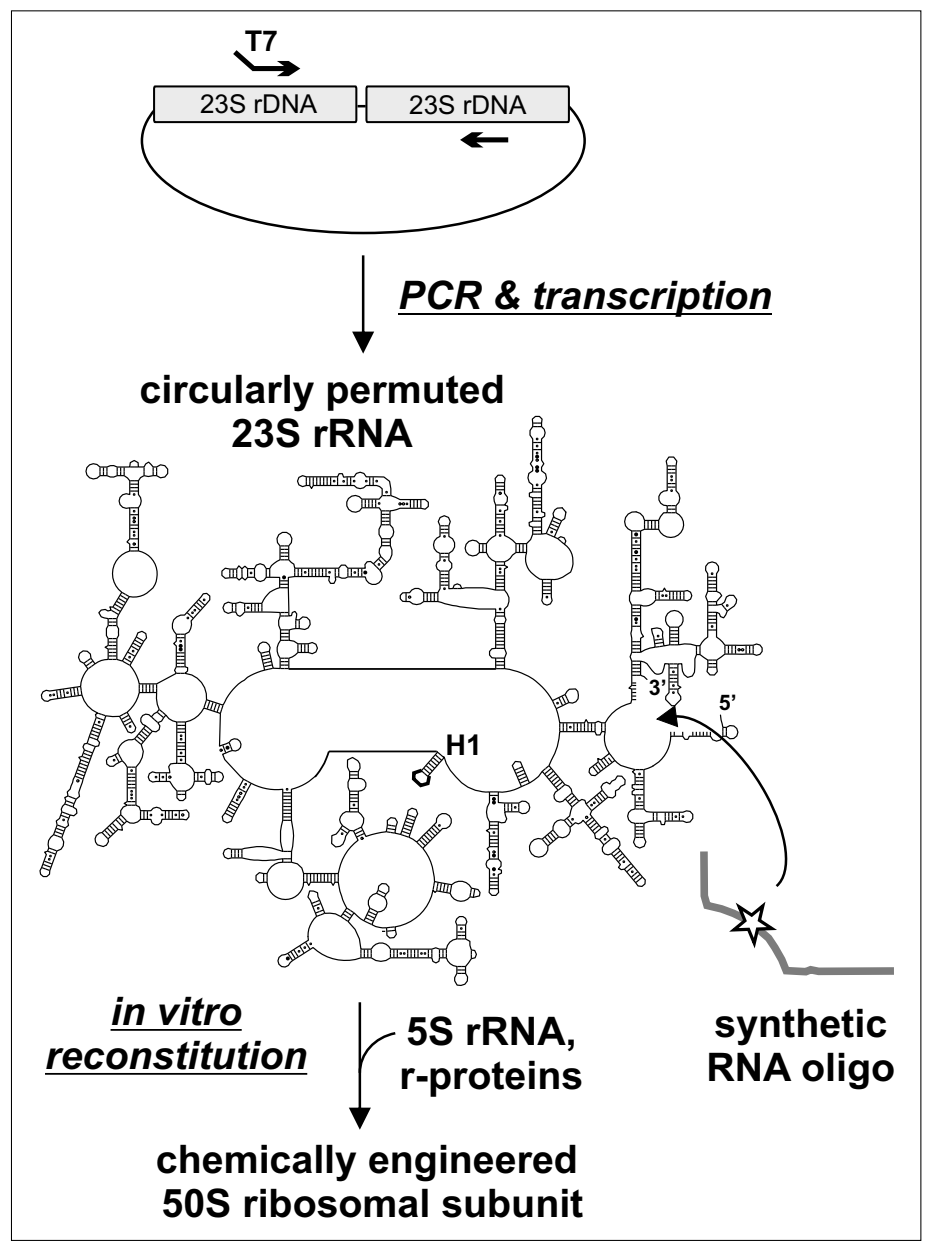

Fig. 1. Experimental design of the 'atomic mutagenesis' approach. The circularly permuted (cp) $23 \mathrm{~S}$ rRNA (schematic secondary structure is shown in the center) is produced by T7 RNA polymerase in vitro transcription from the $\mathrm{cp}-23 \mathrm{~S}$ rDNA template. The $\mathrm{cp}-23 \mathrm{~S}$ rDNA is generated by PCR (PCR primers are depicted as arrows; the forward primer introduces the T7 RNA polymerase promoter) using a plasmid carrying tandemly repeated $23 S$ rRNA genes from $T$. aquaticus. The $\mathrm{cp}-23 \mathrm{~S}$ rRNA is designed so that the natural ends of 23S rRNA, which form helix $1(\mathrm{H} 1)$ in the native $50 \mathrm{~S}$ subunit, are covalently connected (bold black line) and new 5' and 3' ends have been introduced. Thereby a short sequence gap is generated encompassing the residue under investigation. The compensating synthetic RNA oligo (grey) that carries the desired non-natural nucleoside (asterisk) is used in combination with the remaining components of the 50S subunit (5S rRNA and the total r-proteins of the large subunit of $T$. aquaticus) to generate chemically engineered $50 \mathrm{~S}$ subunits by in vitro reconstitution.

tional GTPases, ${ }^{[29,30]}$ and tRNA translocation. ${ }^{[31]}$ One of the most surprising results of our recent research was the finding that a single rRNA backbone group, namely the ribose 2'-OH of the inner core PTC residue A2451, rather than any of the universally conserved nucleobases is crucial for peptide bond synthesis (Fig. 2). Introducing different modifications at this ribose 2' position with markedly different hydrogen donor and acceptor characteristics demonstrated that the 2'-OH group at A2451 donates a proton during the course of amid bond synthesis. ${ }^{[28]}$ The data obtained so far with this approach has enabled us to put forward a novel catalytic mechanism for peptide bond synthesis (Fig. 3) that embraces also relevant data from other research groups. ${ }^{[32-37]}$

Does this mean that the PTC nucleobases are not crucial for ribosome functions? The answer is most likely no, since typically universal conservation indicates functional relevance. While it seems that rRNA backbone groups drive ribosome catalysis ${ }^{[15,28]}$ PTC nucleobases are crucial for tRNA translocation. Employing the atomic mutagenesis approach we were able to identify the importance of a non-Watson-Crick base pair between the two active site residues A2450-C2063 for tRNA translocation. [31] In addition, we identified a functional group at an adenosine at position $\mathrm{A} 2660$ of the $23 \mathrm{~S}$ rRNA to be pivotal for activating the GTPase activity of EF-G (Fig. 2). ${ }^{29,30]}$ A2660 resides in the so-called sarcin-ricin loop (SRL) which has been shown previously by biochemical and structural studies (reviewed in ref. [30]) to be in immediate proximity to the GTP bound in the active site of translational GTPases such as EF-G or EF-Tu. Our atomic mutagenesis studies highlighted the C6 exocyclic amino group at A2660 as a potential trigger of EF-G GTPase activity (Fig. 2). Since the chemical nature of this C6 exocyclic group does not seem to play a critical role (Fig. 2), we interpreted our data such that this nucleobase "communicates' with the G-domain of the elongation factor via $\pi$-stacking interactions of this adenine with amino acid residues of EF-G. Alternatively, the exocyclic N6 amino group could be necessary to sterically clash into EF-G residues at the $\mathrm{G}$ domain. These interactions may act as the trigger to induce conformational changes within the $\mathrm{G}$ domain of EF-G leading to GTPase activation. Due to the universal conservation of the SRL and the G-domains of translational GTPases, this might be a commonly used GTPase activation mechanism among all translational GTPases, such as initiation factor 2 (IF2), EF-Tu, EF4 (LepA) or release factor 3 . Indeed all of these translational GTPases have been shown to equally depend on the presence of this 


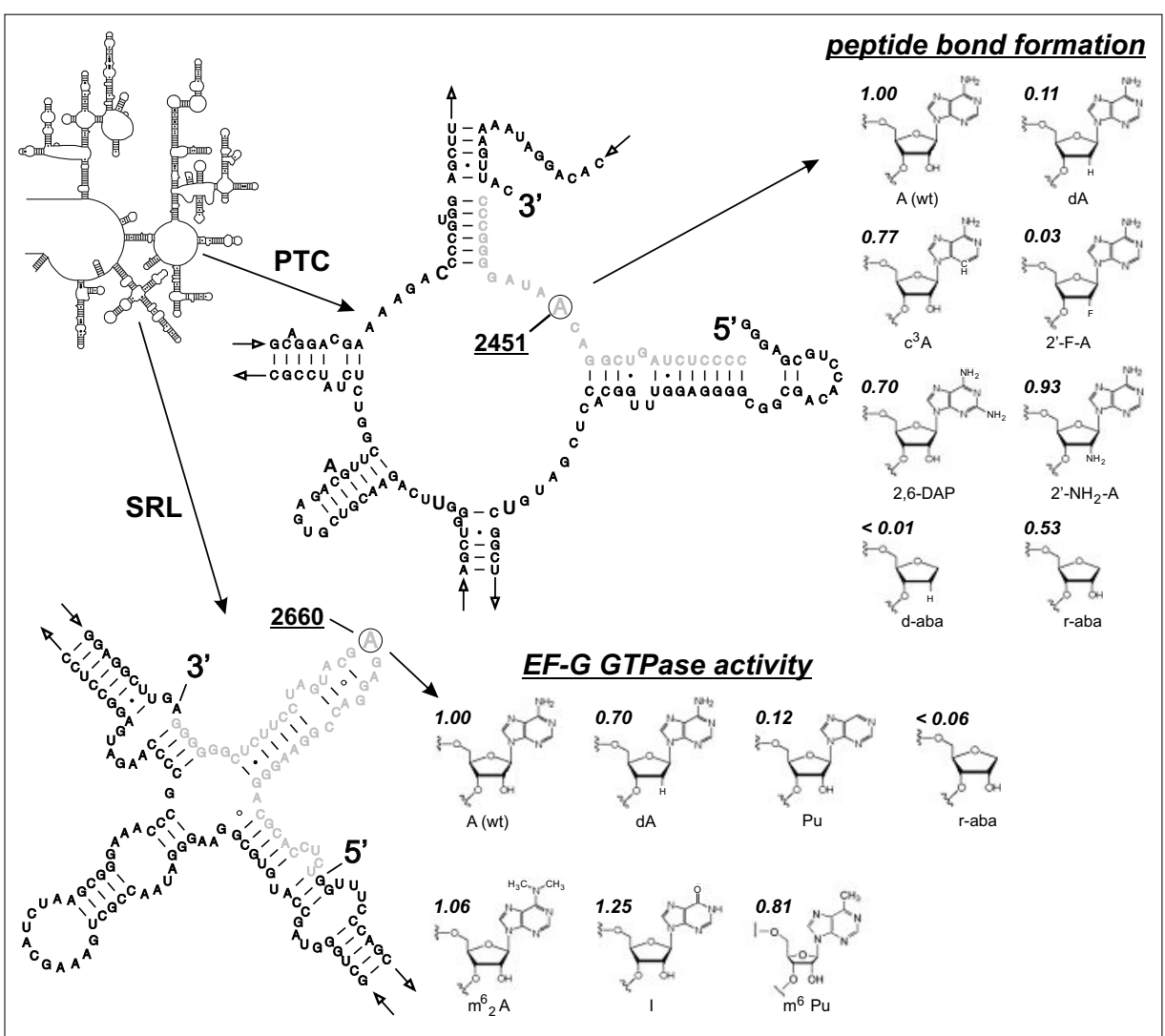

Fig. 2. Atomic mutagenesis at A2451 in the peptidyl transferase center (PTC) and at A2660 in the sarcin-ricin-loop (SRL) of 23S rRNA. Secondary structures of the respective active sites are shown whereas the newly introduced endpoints of the cp-23S rRNA constructs are shown ( $5^{\prime}$ and 3', respectively). The chemically synthesized RNA oligos, which compensate for the missing rRNA fragments, are shown in grey and are held in place by Watson-Crick base pairing with the $\mathrm{cp}-23 \mathrm{~S}$ rRNAs. The chemical structures of nucleoside analogs that were introduced at A2451 or A2660 are depicted and the relative rates of peptide bond formation ${ }^{[2,28]}$ or EF-G GTPase activity ${ }^{[29]}$ are shown for each nucleoside analog above the structures. The rates were normalized to reconstituted ribosomes containing the synthetic wild-type RNA fragment. Tested nucleoside analogs: dA: 2'-deoxy-adenosine; c ${ }^{3}$ A: 3-deaza-adenosine; 2'-F-A: 2'-fluoro-adenosine; 2,6-DAP: 2,6-diaminopurine; 2'- $\mathrm{NH}_{2}-\mathrm{A}$ : 2'-amino-adenosine; d-aba: 2'-deoxy-ribose-abasic analog; r-aba: riboseabasic analog; Pu: purine; $m_{2}^{6} A$ : N6,N6-dimethyladenosine; I: inosine; $m^{6} \mathrm{Pu}$ : 6-methylpurine.

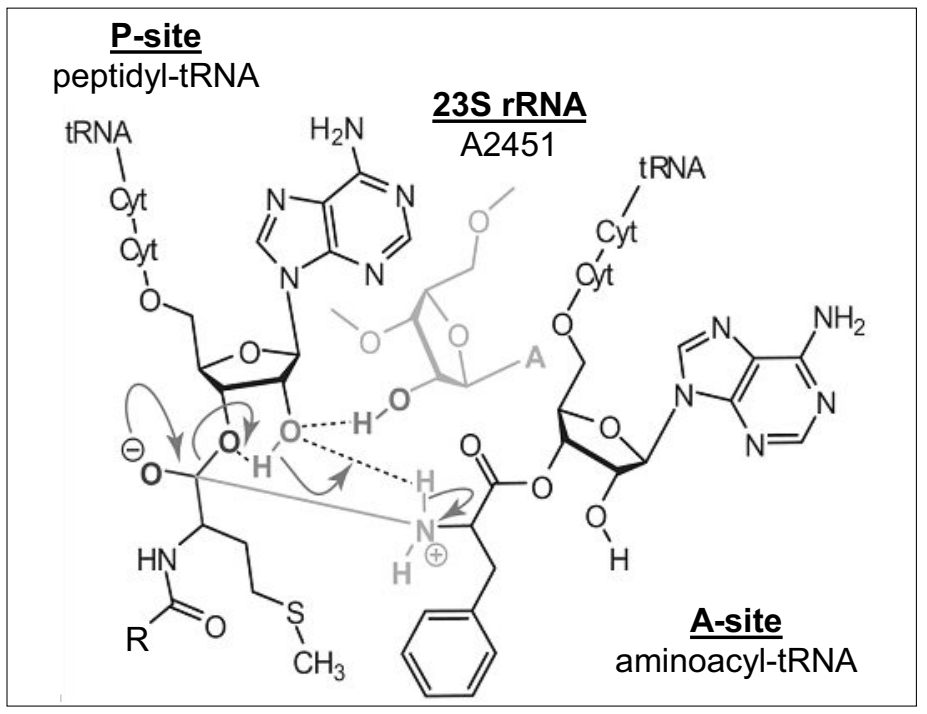

Fig. 3. Model for the mechanism of peptide bond formation proposing a role for A2451 based on our experimental finding that its hydrogen donor propensity is a stringent requirement for activity. ${ }^{[28}$ The A2451 2'-O-H ••• O(2') A76 hydrogen bond assists in P-site tRNA A76 ribose positioning in its functionally competent confirmation and in suppression of spontaneous intra-molecular transesterification. In this model the nucleophilic attack of the $\alpha$-amino group of A-site bound aminoacyl-tRNA on the ester carbonyl carbon is accompanied by a concomitant acceptance of a proton from the $\alpha$-amino group by the A76 2'-O of the peptidyl-tRNA which simultaneously donates its proton to the vicinal 3 '-O. Arrows indicate pair-wise electron movement for proton shuttling after the attack of the $\alpha$-amino nucleophile has established the tetrahedral intermediate.
C6 exocyclic group at A2660. ${ }^{[30]}$ Structural data suggest an alternate or additional ribosomal trigger to fire GTPase activity on ribosome-bound elongation factors, namely a non-bridging oxygen at the phosphordiester bond between SRL residues G2661 and A2662. ${ }^{[38]}$ Equipped with the atomic mutagenesis approach this model can now be tested experimentally since it enables us to individually manipulate the suspected rRNA backbone groups in that region of the SRL.

\section{Research Directions and Perspectives}

Initial proof of principle experiments demonstrated that unprecedented molecular insight can be gained by applying the atomic mutagenesis approach. However in these initial applications of the technique the chemically engineered ribosomes were used in simplified model reactions of protein synthesis primarily under single turnover reaction conditions (e.g. peptide bond formation, pept-tRNA hydrolysis). However, protein biosynthesis by the ribosome is a very dynamic multi-functional and iterative process, thus it is possible that functionally crucial $23 \mathrm{~S}$ rRNA groups have been overlooked in these initial studies. In order to improve the biological relevance of the assays and to challenge chemically engineered ribosomes in a more physiologically relevant system, we have recently optimized reaction conditions to employ these in vitro generated particles in genuine in vitro translation reactions. And indeed chemically engineered ribosomes turned out to be active in poly(U)-directed poly(Phe) synthesis and, more importantly, also in translation reactions using natural mRNA templates (Fig 4). ${ }^{[25]}$ Currently we use the atomic mutagenesis approach to (i) study the function of the 50S E-site, (ii) to 'retro-evolve' a miniaturized functional ribosome, and (iii) to learn more about the regulatory potential of interactions between the growing peptide chain, as it leaves the PTC, and the nascent peptide exit tunnel wall in the large ribosomal subunit.

The experimental evidence gathered to date with the atomic mutagenesis technique open doors for novel avenues of biochemical research and revealed the ribosome, the largest known ribozyme, as being amenable to synthetic biology approaches. ${ }^{[23]}$ It appears possible that the chemical characteristic of the ribosome can be rationally engineered utilizing the 'atomic mutagenesis' approach to generate an in vitro translation system that allows the synthesis of 'designer peptides' carrying, for example, several consecutive unnatural amino acids. 


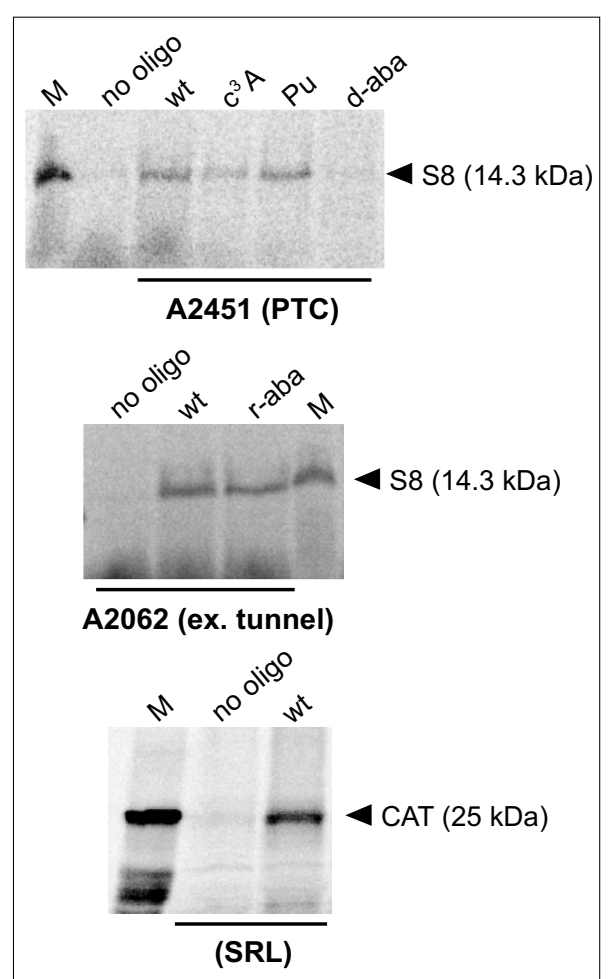

Fig. 4. Chemically engineered ribosomes are active in in vitro translation. In vitro assembled ribosomes, programmed with mRNAs encoding either r-protein S8 or the enzyme chloramphenicol acetyltransferase, are capable of translating full-length $\left[{ }^{35} \mathrm{~S}\right]$-labeled proteins (arrow heads). The reaction products were separated via SDS polyacrylamide gel electrophoresis. Results with three different $\mathrm{cp}-23 \mathrm{~S}$ rRNA constructs are shown that allow distinct $50 S$ active sites to be investigated by the atomic mutagenesis approach (PTC: peptidyl transferase center; ex. tunnel: nascent peptide exit tunnel; SRL: sarcin-ricin-loop). Also in this assay the requirement for a ribose 2'-OH group at A2451 is evident (top gel). Lanes labeled with ' $M$ ' show an aliquot of an in vitro translation reaction using native $70 S$ ribosomes from E. coli and serve as length markers. For abbreviation of nucleoside analogs see Fig. 2.

\section{Acknowledgements}

The author thanks his past and present coworkers and collaboration partners for realizing these studies. The work outlined above was financially supported by the Swiss National Foundation (31003A_143388), the Austrian Science Foundation (grants Y315, P16932, P18709), the Austrian Genome Research Program GenAU (D-110420-012-012), and the University of Bern.

Received: March 25, 2013
[1] M. D. Erlacher, N. Polacek, RNA Biol. 2008, 5, 5.

[2] N. Polacek, A. S. Mankin, Crit. Rev. Biochem. Mol. 2005, 40, 285.

[3] K. H. Nierhaus, H. Schulze, B. S. Cooperman, Biochemistry Int. 1980, 1, 185.

[4] A. Sievers, M. Beringer, M. V. Rodnina, R. Wolfenden, Proc. Natl. Acad. Sci. USA 2004, 101, 7897.

[5] N. Ban, P. Nissen, J. Hansen, P. B. Moore, T. A. Steitz, Science 2000, 289, 905.

[6] P. Nissen, J. Hansen, N. Ban, P. B. Moore, T. A. Steitz, Science 2000, 289, 920.

[7] D. N. Wilson, G. Blaha, S. R. Connell, P. V. Ivanov, H. Jenke, U. Stelzl, Y. Teraoka, K. N. Nierhaus, Cur. Prot. \& Pept. Sci. 2002, $3,1$.

[8] N. Demeshkina, L. Jenner, E. Westhof, M. Yusupov, G. Yusupova, Nature 2012, 484, 256.

[9] J. M. Ogle, V. Ramakrishnan, Аnпи. Rev. Biochem. 2005, 74, 129.

[10] I. Wohlgemuth, C. Pohl, M. V. Rodnina, EMBO J. 2010, 29, 3701.

[11] M. Johansson, E. Bouakaz, M. Lovmar, M Ehrenberg, Mol. Cell. 2008, 30, 589 .

[12] V. I. Katunin, G. Muth, S. A. Strobel, W. Wintermeyer, M. V. Rodnina, Mol. Cell 2002, 10,339 .

[13] W. Szaflarski, O. Vesper, Y. Teraoka, B. Plitta, D. N. Wilson, K. H. Nierhaus, J. Mol. Biol. 2008, 380, 193

[14] H. S. Zaher, R. Green, Cell 2009, 136, 746.

[15] M. Amort, B. Wotzel, K. Bakowska-Zywicka, M. D. Erlacher, R. Micura, N. Polacek, Nucleic Acids Res. 2007, 35, 5130.

[16] N. Polacek, M. G. Gomez, K. Ito, Y. Nakamura, A. S. Mankin, Mol. Cell 2003, 11, 103.

[17] M. Laurberg, H. Asahara, A. Korostelev, J. Zhu, S. Trakhanov, H. F. Noller, Nature 2008, 454, 852.

[18] M. D. Erlacher, N. Polacek, Biotechnologia 2009, 1,65

[19] Y. Huang, M. Sprinzl, Angew. Chem. Int. Ed. 2011, 50, 7287
[20] M. Simonovic, T. A. Steitz, Biochim. Biophys. Acta. 2009, 1789, 612 .

[21] H. S. Zaher, J. J. Shaw, S. A. Strobel, R. Green, EMBO J. 2011, 30, 2445.

[22] M. Koch, Y. Huang, M. Sprinzl, Angew. Chem. Int. Ed. 2008, 47, 7242.

[23] A. Chirkova, M. D. Erlacher, R. Micura, N. Polacek, Curr. Org. Chem. 2010, 14, 148.

[24] N. Polacek, M. Gaynor, A. Yassin, A. S. Mankin, Nature 2001, 411, 498.

[25] M. D. Erlacher, A. Chirkova, P. Voegele, N. Polacek, Nature Prot. 2011, 6, 580.

[26] M. D. Erlacher, K. Lang, N. Shankaran, B. Wotzel, A. Huttenhofer, R. Micura, A. S. Mankin, N. Polacek, Nucleic Acids Res. 2005, 33,1618 .

[27] M. D. Erlacher, K. Lang, B. Wotzel, R. Rieder, R. Micura, N. Polacek, J. Am. Chem. Soc. 2006, $128,4453$.

[28] K. Lang, M. D. Erlacher, D. N. Wilson, R. Micura, N. Polacek, Chem. Biol. 2008, 15, 485.

[29] N. Clementi, A. Chirkova, B. Puffer, R. Micura, N. Polacek, Nature Chem. Biol. 2010, 6, 344.

[30] N. Clementi, N. Polacek, RNA Biol. 2010, 7, 521.

[31] A. Chirkova, M. D. Erlacher, N. Clementi, M. Zywicki, M. Aigner, N. Polacek, Nucleic Acids Res. 2010, 38, 4844.

[32] P. Bieling, M. Beringer, S. Adio, M. V. Rodnina, Nat. Struct. Mol. Biol. 2006, 13, 423.

[33] S. Dorner, C. Panuschka, W. Schmid, A. Barta, Nucleic Acids Res. 2003, 31, 6536.

[34] T. M. Schmeing, K. S. Huang, S. A. Strobel, T. A. Steitz, Nature 2005, 438, 520.

[35] S. Trobro, J. Aqvist, Proc. Natl. Acad. Sci. USA 2005, 102, 12395.

[36] J. S. Weinger, K. M. Parnell, S. Dorner, R. Green, S. A. Strobel, Nat. Struct. Mol. Biol. 2004, 11, 1101.

[37] A. Yonath, Biol. Chem. 2003, 384, 1411.

[38] R. M. Voorhees, T. M. Schmeing, A. C. Kelley, V. Ramakrishnan, Science 2010, 330, 835.

\author{
Received. March 25, 2013
}

